\title{
Microwaves, Power Ultrasound, and lonic Liquids. A New Synergy in Green Organic Synthesis
}

\author{
Jean-Marc Lévêque ${ }^{\mathrm{a}}$ and Giancarlo Cravotto*b
}

\begin{abstract}
Although the vast majority of organic chemists still cling to conductive heating as a means to promote reactions, major advances have recently been made in this connection. Both dielectric microwave heating (MW) and power ultrasound (US) are being increasingly exploited in organic synthesis, and their combined use is one of the most promising innovations. A long way from pioneering approaches, when domestic MW ovens and US cleaning baths were poorly standardized tools, these techniques now appear in reproducible, high-yield synthetic protocols. Their important contributions towards developing environment-friendly procedures overlap the application domain of room-temperature ionic liquids (RTILs). The synthesis itself of these highly versatile green solvents can be efficiently promoted under US and/or MW irradiation. This review focuses on the advantages arising from the use of these energy sources and their combination with unconventional reaction media such as RTILs. The synergies arising from the combined use of US, MW and RTIL will certainly go a long way to meet the increasing demand for environmentally benign chemical processes.
\end{abstract}

Keywords: Combined irradiation · Ionic liquids · Microwaves · Power ultrasound · Synthesis

\section{Introduction}

Because of the irreplaceable roles they play in many different physical and chemical processes, both microwaves (MW) and power ultrasound (US) have seen their range of application expand enormously. In many instances their effects have been comparatively measured in the same system and a synergic effect has been observed when US and MW were used in combination [1]. Although their application domains overlap only partially, they both have much to contribute to green chemistry, particularly

\footnotetext{
${ }^{\star}$ Correspondence: Prof. G. Cravotto ${ }^{b}$

Tel.: + 390116707684

Fax: + 390116707687

E-Mail: giancarlo.cravotto@unito.it

aLaboratoire de Chimie Moléculaire et Environnement ESIGEC

Université de Savoie

73376 Le Bourget du Lac

France

E-Mail: Jean-Marc.Leveque@univ-savoie.fr

bDipartimento di Scienza e Tecnologia del Farmaco

Università di Torino

Via Giuria 9

10125 Torino Italy
}

towards developing environment-friendly synthetic procedures. MW and US have also emerged as powerful techniques for the degradation of persistent organic pollutants (POPs) [2], for the regeneration of industrial adsorbents [3], in the extraction of plant materials [4], for histological preparations [5], pesticide recovery, [6] and in the production of nanomaterials [7].

MW [8][9] and US [10] are being increasingly exploited in organic synthesis (mostly on the laboratory scale so far) because they bring reactions to completion in minutes or even seconds rather than hours or days. They also can induce reactions that would otherwise prove very laborious, enhance heterogeneous catalysis [11] and even bring out unusual chemoselectivities, thus opening up new synthetic pathways. They can be applied in a straightforward manner to some, but certainly not all, reactions; indeed, painstaking preliminaries are often required to identify optimal conditions that often turn out to be quite different from traditional ones. Recent publications report a variety of straightforward synthetic laboratory-scale procedures, carried out in parallel under MW and US [12-14]; much work is under way for their scale-up, no easy task by any means [15-18]. The MW/US combination has been introduced in organic synthesis [10] and in analytical chemistry [19] to facilitate sample prepa- ration, although technical hurdles are still hampering a widespread use.

MW- and US-promoted reactions have been successfully carried out in non-conventional media such as room-temperature ionic liquids (RTIL), revealing a host of interesting synergic effects and resulting in marked advantages from the standpoint of green chemistry. Throughout the past decade the number of publications concerning ionic liquids has grown exponentially. The acronym RTIL, no longer a new-fangled symbol in the academic and industrial scientific communities, refers to a thermally stable compound melting below $100{ }^{\circ} \mathrm{C}$ that is made up of a bulky organic cation in combination with an inorganic or organic anion. The use of these efficient, versatile (even chiral) and recyclable media for chemical reactions is now one of the pillars of green chemistry. Moreover, their recently discovered use in the dual solvent/catalyst role is a fast-growing area of research.

\section{Combined MW/US Irradiation}

Simultaneous irradiation with MW and US is a very promising innovation. Especially in the field of heterogeneous catalysis [11] additional effects are to be expected when dielectric heating is associated with the large amount of energy released in 
cavitational collapse (causing particle fragmentation and molecular excitation). This combination can promote or improve many chemical and physical processes [1] such as synthesis, extraction of natural matrices, and preparation of analytical samples.

Simultaneous irradiation in a single reaction vessel can be achieved in a MW oven where the horn (made of quartz, pyrex or ceramic material) is directly inserted. The following pictures show a modified domestic oven (Fig. 1) and a professional multimode oven (Fig. 2), two devices currently employed in our laboratories.

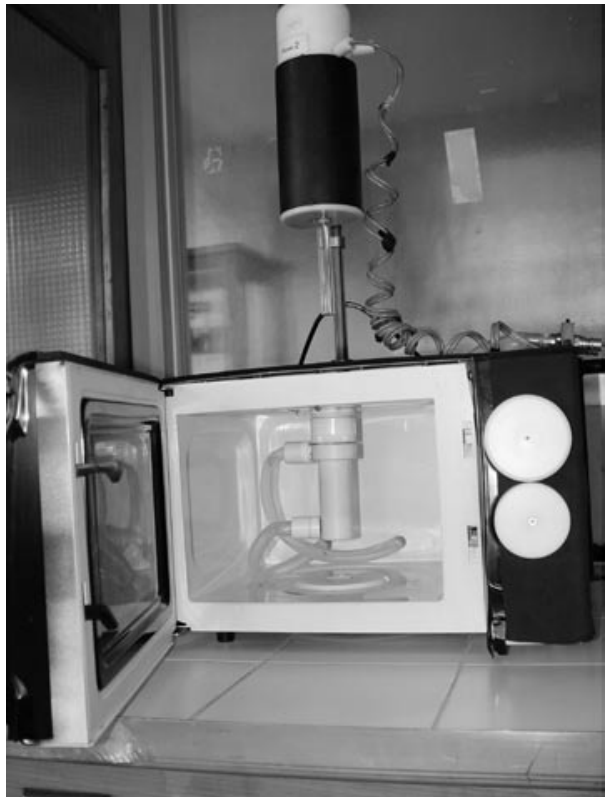

Fig. 1. Reactor featuring simultaneous MW/US irradiation (fused-quartz horn)

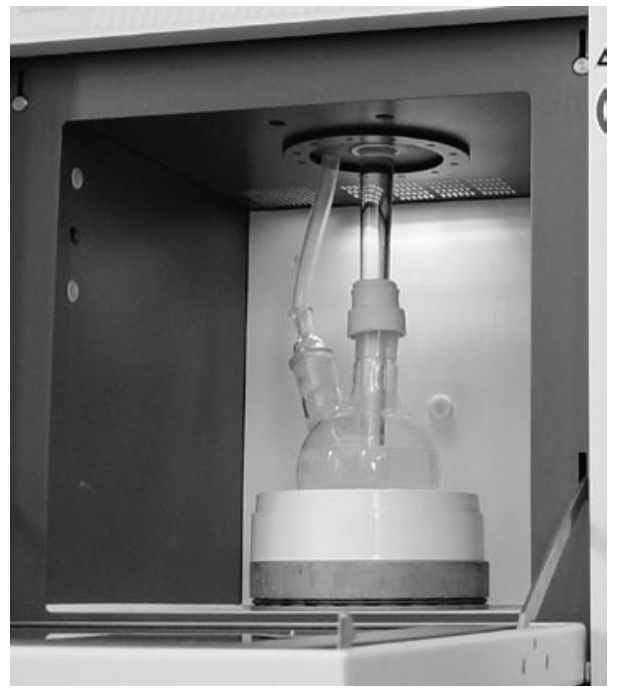

Fig. 2. Professional multimode MW oven equipped with a pyrex horn for simultaneous MW/US irradiation

Peng and Song [20] first built a pioneering device by inserting a detachable horn (of unspecified material) in a modified domestic MW oven; radiation leakage
Table 1. Hydrazinolysis of methyl salicylate using different methods

\begin{tabular}{lll} 
Method & Time & Yield [\%] \\
\hline Conventional reflux & $9 \mathrm{~h}$ & 73 \\
US (50 W) reflux & $1.5 \mathrm{~h}$ & 79 \\
MW (200 W) & $18 \mathrm{~min}$ & 80 \\
US (50 W) / MW (200 W) & $40 \mathrm{~s}$ & 84 \\
a Isolated yields & &
\end{tabular}

was prevented by a metal mesh screen fastened tightly onto the horn. Simultaneous irradiation dramatically sped up hydrazinolysis of esters in the absence of organic solvents and other auxiliary agents (Table $1)$. The authors attempted to explain this success on the basis of enhanced heat and mass transfer.

The same authors applied their technique to the Williamson ether synthesis, doing away with phase-transfer catalysts [21], and to the Knoevenagel-Doebner reaction in aqueous media [22]. The latter, a simple, efficient procedure for the synthesis of 3-aryl acrylic acids from the corresponding aromatic aldehydes, avoided the use of organic solvents and allowed the catalyst to be easily recovered and reused.

In an alternative, less efficient setup that avoided subjecting the horn to the high-frequency field, a low-viscosity apolar liquid (decaline), exposed to US outside the MW oven, conveyed the former radiation through a double-jacketed pyrex vessel to the reacting mixture placed in the oven itself. Simultaneous MW/US irradiation was first described by Chemat and coworkers [23][24] who used this device for the esterification of stearic acid with butanol under heterogeneous catalysis, reporting improved results in comparison with the MW-promoted reaction. By simultaneous irradiation they also could successfully digest at atmospheric pressure solid and liquid samples for chemical analysis and called their method 'US-assisted MW digestion' [25].

All these results portended great advances in heterogeneous organic synthesis, also from the environmental point of view.

Finally, in the sequential irradiation method that allows the use of commercially available metal horns, a pump circulates the reacting mixture through two separated reaction cells (See the flow reactor in Fig. 3 and 4).

With a series of synthetically useful aryl-aryl couplings (catalyzed by $\mathrm{Pd} / \mathrm{C}$ ) the last system gave better yields and shorter reaction times than did individual irradiations with either US or MW (Table 2) [26]. Neither phosphine ligands nor phasetransfer catalysts were required. Likewise

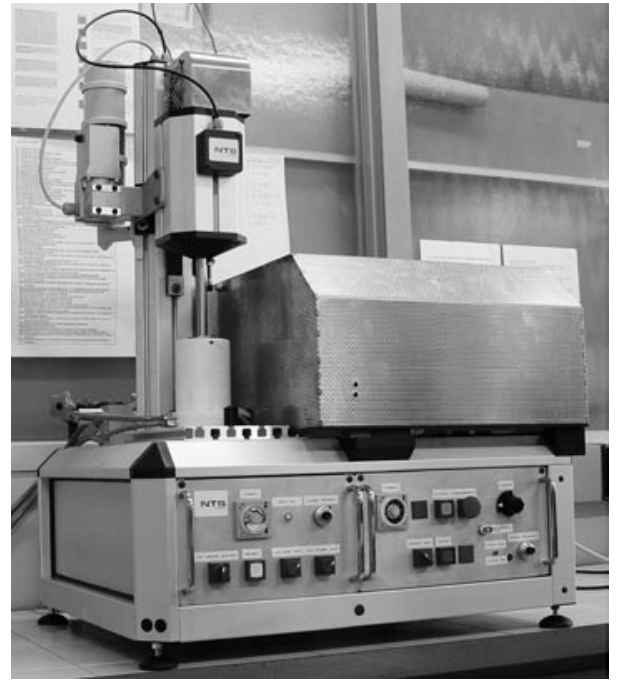

Fig. 3. Flow reactor combining MW and US irradiation

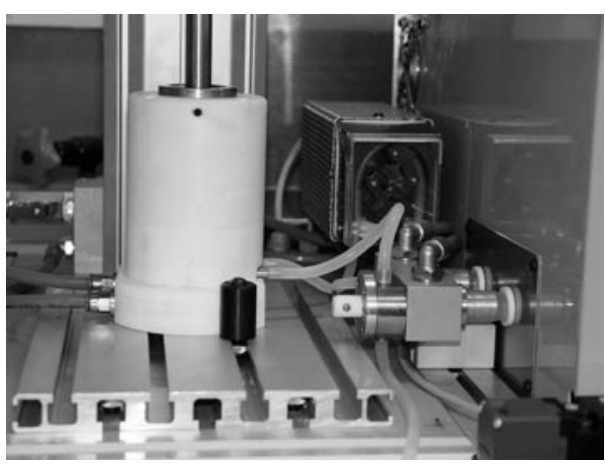

Fig. 4. Peristaltic pump and connection tubing in the MW/US flow reactor

homo- and cross-couplings of arylboronic acids and aryl halides were further improved using the combined flow reactor (Table 2). Electron-deficient aryl chlorides also reacted when palladium(II) acetate was employed as catalyst.

The most recent successful application of the method was the synthesis of $\beta$-aminoketones via Mannich reaction in water (Scheme 1) [27]. With combined irradiation the reaction time was reduced to less than 1 min, 10-30 fold less than under irradiation with either MW or US. 
Table 2. Compared yields of Suzuki reactions under US or MW alone and combined MW/US in the flow reactor.

\begin{tabular}{lllll} 
aryl halide & boronic acid & $\begin{array}{l}\text { US } \\
\text { Yield [\%] }\end{array}$ & $\begin{array}{l}\text { MW } \\
\text { Yield [\%] }\end{array}$ & $\begin{array}{l}\text { Combined } \\
\text { US/MW } \\
\text { yield [\%] }\end{array}$ \\
\hline 3-bromoanisole & phenylboronic & 54 & 64 & 88 \\
2-iodothiophene & phenylboronic & 40 & 37 & 59 \\
4-chloro-nitrobenzene & phenylboronic & 22 & 30 & 57 \\
- & thianthrene-1-boronic & 48 & 55 & 69 \\
- & 4-t-butylboronic & 68 & 74 & 86
\end{tabular}

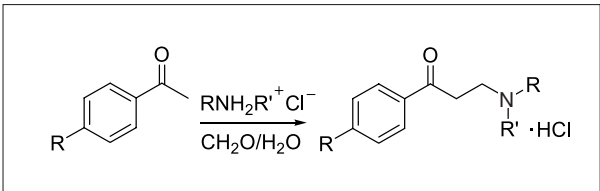

Scheme 1. Mannich reaction under combined MW/US irradiation

\section{Room Temperature Ionic Liquids}

Organic cations can be obtained by extending the basic valences of nitrogen, sulphur or phosphorus, although nitrogenbased RTILs containing a dialkylimidazolium or an alkylpyridinium cation are the most commonly used and commercially available ones. Several anions, whether organic or inorganic, are combined with them, the most frequently used being $\mathrm{Cl}^{-}$, $\mathrm{Br}^{-}, \mathrm{AlCl}_{4}^{-}, \mathrm{BF}_{4}^{-}, \mathrm{PF}_{6}^{-}, \mathrm{OTf}^{-}$, and $\mathrm{N}(\mathrm{Tf})_{2}^{-}$[28] (Scheme 2).

Because of their remarkable and possibly 'tunable' physico-chemical properties, these compounds attract a growing interest as environmentally benign media that have proven suitable for carrying out Diels-Alder [29], Friedel-Craft [30], Heck [31],
Suzuki [32], Mannich-type reactions [33], Michael additions [34], oxidations [35], hydrogenations, [36] and so forth. Originally introduced in electrochemistry, RTILs are now being tested in various applications for a number of physico-chemical processes such as extraction [37], dissolution [38], (bio)catalysis [39], polymerization [40], and even to replace thermal oils in the production of solar energy [41]. Basically, RTILs combine to a remarkable extent the properties of both molecular solvents and molten salts. Being non-flammable and having barely measurable vapour pressures, they can be efficiently recycled and may be the answer to the problems of VOC. Their ability to dissolve high concentrations of non polar as well as polar compounds confers on them a versatility as reaction media that traditional molecular solvents could never approach [42]. Moreover, by appropriately varying the choice of anion, the kind of organic cation and of its substituent alkyl chains (possibly bearing functional groups), we can finely tune the melting point, density, viscosity, solvent power for specific organic moieties and water character of an RTIL. Consequently, we consider able' and speak of 'solvents design', i.e. the their physico-chemical properties as 'tun-

process of obtaining a TSIL (Task-Specific Ionic Liquid). By this acronym we mean a particular RTIL bearing the appropriate functional groups that will confer on it the desired physico-chemical properties or reactivity. For example, in the field of asymmetric synthesis, chiral RTILs have been developed to induce enantioselectivity in a chemical reaction [43]. Most of them are derived from the 'chiral pool' [44]. Even if results have not, so far, matched expectations, in principle the use of a chiral, recyclable solvent may become the most convenient method to make asymmetric compounds from non-chiral starting materials.

\subsection{Preparation of RTILs under MW Irradiation}

Two distinct steps are necessary to obtain RTILs. In the first one (Mentschukin reaction) the valence extension of the heteroatom yields the precursors of ionic liquids [45], i.e. their halides (first-generation RTILs). The second step (Finkelstein reaction) consists in the metathesis of the halide counter-anion that leads to the second generation of ionic liquids [46], containing bulkier anions and having more interesting properties such as lower melting points or choice in the water character. Although the process is not beset by any major problem, it is notoriously time-consuming. As a typical example, the synthesis of 1-butyl-3-methylimidazolium chloride takes as long as 72 $\mathrm{h}$; then the metathesis with a bulkier anion takes an additional 24-48 h, if purification prior to use is included [47]. Overall required time thus amounts to 5-7 d (Scheme 3 ), an unbearable delay for industry.

Although several attempts were made in the late nineties to improve the access to second-generation RTILs using different reagents, none of them really conformed to the principles of green chemistry. Moreover, the purification steps for the removal of halide impurities and water remain a troublesome hindrance.

A direct and efficient way to speed up the preparation of RTILs has been provided by non-conventional activation methods, namely MW and US technologies. Their adoption has been spurred by the same emergence of green chemistry that brought to the fore new solvents and reaction media, such as supercritical fluids and ionic liquids. MW is now established as an efficient tool by which reaction times can be cut down by several orders of magnitude and yields considerably increased [48]. Owing to their ionic nature, ionic liquids absorb of course MW irradiation.

In a preliminary report, Varma et al. prepared several 1,3-dialkylimidazolium halides working in an open vessel under continuous MW irradiation with an excess of alkyl halide [49]. The preparation time was cut down from several hours to a few 


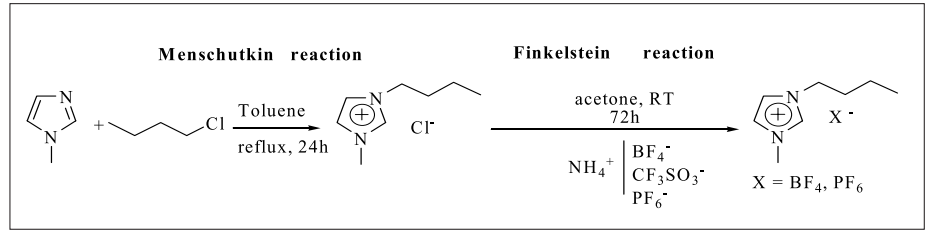

Scheme 3. Two-step synthesis of RTILs under conventional heating

minutes, although the purity of the products left much to be desired. The same authors noted in a subsequent article that continuous irradiation may result in overheating and thus lead to coloured impurities. The use of an open vessel with volatile alkyl halides can be hazardous; moreover, because 1-methylimidazole is highly hygroscopic, during the process water was inevitably absorbed, which was difficult to remove under vacuum. Rebeiro et al. improved the process by employing a closed vessel, which raised the scale up to $50 \mathrm{~g}$ and allowed the use of methylimidazole or pyridine as precursors of the organic cations together with chloride or bromide alkyls [50]. They observed that the higher the temperature (and consequently the pressure) inside the vessel, the shorter the necessary reaction time. Finally, a stoichiometric amount of both starting materials could be used with no apparent yield loss. This process highlights several aspects of green chemistry as it used no solvent, took a very short time (saving energy) and had a high atom efficiency. In a systematic study on the synthesis of nitrogen-based heterocycles, Seddon et al., together with an impressive number of alkyl halides, presented a general MW-assisted method for a medium-scale access to the most commonly used ionic liquids [51]. Varma et al. went on to elaborate the first MW-assisted preparation of second-generation RTILs containing the bulky tetrafluoroborate anion [52] (Scheme 4).

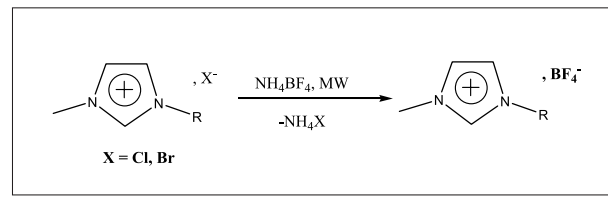

Scheme 4. MW-assisted preparation of tetrafluoroborate-based RTIL

They noticed that at elevated power levels a partial decomposition of the material, presumably the ammonium salt, occurred (probably due to local overheating) which led to a lower yield. To circumvent this hurdle, they intermittently irradiated the system at moderate power levels with stirring to avoid local hot spots. A number of RTILs and TSILs have since been prepared by a MW-assisted procedure [53-57]; a few examples are given in Scheme 5:

\subsection{Preparation of RTILs under Ultrasonic Irradiation}

The preparation of RTILs under ultrasonic irradiation has been studied to a lesser extent. Besides generating highly reactive intermediates that may change the mechanistic pathway, cavitation in heterogeneous systems also causes physical changes such as micro-emulsion, mass transport, and reduction of particle size. Ultrasonic irradiation can therefore be particularly advantageous with heterogeneous systems, replacing the use of a phase transfer agent. Varma et al. tried to obviate the high viscosity of RTILs by preparing the halide precursors in a neat system under ultrasonic irradiation in a closed vessel [58]. Expecting no change in the mechanistic pathway as the Menschutkin step involves an ionic pathway, the authors just resorted to US for an efficient mixing and for heat supply, both effects coming from the cavitation phenomenon. When 1-methylimidazole and alkyl halides such as chlorobutane or bromobutane are mixed together, the resulting system is homogeneous. However, as the ionic liquid starts to form, a second phase appears. Because of its high viscosity, the resulting heterogeneous system cannot be mixed well by the common stirring devices, increasing reaction time; on the contrary, US mix it very efficiently. Using an ultrasonic bath with bromide and iodide alkyls and an ultrasonic probe for chloride alkyls, the authors achieved a solvent-free approach resulting in shorter reaction times and lower reaction temperatures compared to conventional heating. They also reported that the purity of RTILs prepared in this way was higher than that obtainable with classical methods. To our best knowledge, this is the only paper dealing with the preparation of halide precursors of RTILs under ultrasonic irradiation. Ultrasonic irradiation is even more advantageous to access secondgeneration RTILs with bulkier anions by the Finkelstein step, as here the system is heterogeneous. Lévêque et al. generalized the US-assisted method to obtain a large number of RTILs with dialkylimidazolium, alkylpyridinium and pyrrolidinium moieties. Reaction time was $1 \mathrm{~h}$, compared to 24-48 h required under mechanical stirring, and products were much purer [59]. The authors proved that the dramatic decrease of the reaction time was a direct ultrasonic effect brought about by physical phenomena accompanying cavitation, especially a reduction of particle size which increased greatly the reactive surface area of the solids [60].

\subsection{The Use of RTILs as Green, Recyclable Media in MW-assisted Synthesis}

\subsubsection{Catalysis}

RTILs have proved almost ideally suited to trap, immobilize and preserve from decomposition organic and transition-metal

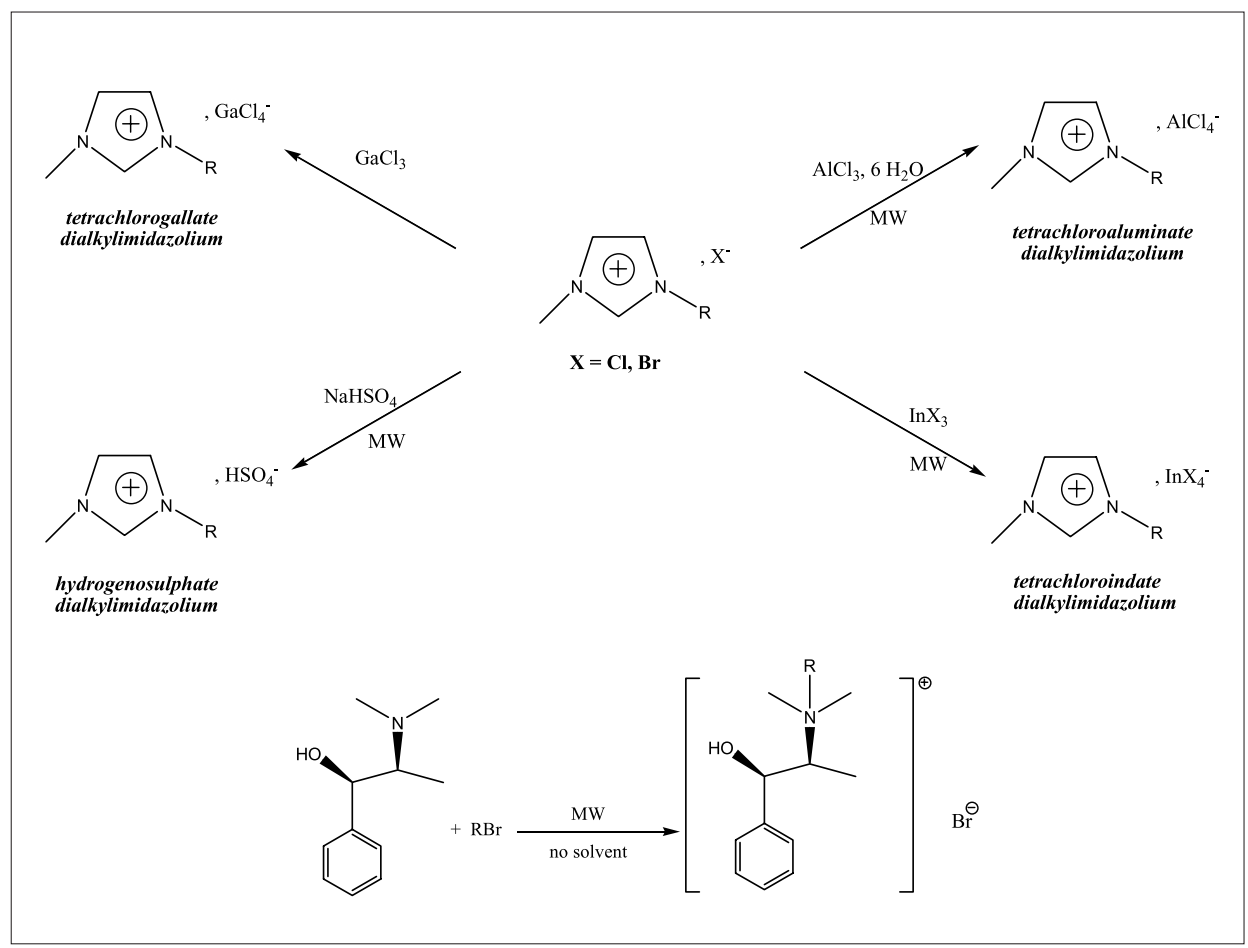

Scheme 5. Selected examples of MW-assisted preparation of TSIL 
catalysts while enhancing reaction rates and selectivities [42]. Moreover, the recycling of these solvent-catalyst systems can be very efficient and cause no loss of activity through several cycles. One of the reactions that has been most thoroughly studied in alternative solvents is the Heck reaction, a Pd-catalyzed $\mathrm{C}-\mathrm{C}$ coupling [31]. It has been widely studied in RTILs under both mechanical stirring plus conventional heating and MW-assisted conditions. It gave good results under conventional heating but usually took long reaction times and/or suffered from recycling problems.

As the ionic nature of RTILs makes them highly absorbing for MW, this method has emerged as a powerful and easily controllable tool for overcoming the excessive length of reaction times. When used under MW in both hydrophobic (BMIMPF ${ }_{6}$ ) and hydrophilic $\left(\mathrm{OMIMBF}_{4}\right)$ RTILs, Heck Pd catalysts gave average-to-good conversions/yields [61] (Scheme 6).

Moreover, in both cases the catalyst could be effectively recycled five successive times without any loss of activity.

The Tsuji-Trost coupling reaction, catalyzed by $\mathrm{Pd}(0)$ species, was also tried under MW irradiation [62]. Over the past years a biphasic water/organic solvent system was introduced as an embryonic green procedure for allylic substitutions, which were still beset with long reaction times and loss of catalyst after a single use. By replacing organic solvents with a hydrophilic ionic liquid and working under MW, Liang et al. obtained good yields in a very short time (Scheme 7).

They could recycle the biphasic solvent system together with the catalyst and reuse it eight times without any loss of activity. This procedure fully embodied a green approach, featuring a clean and easy work-up with a non-hazardous solvent system.

\subsubsection{Combinatorial/Multi-step Synthesis}

The combination of RTILs with MW irradiation also offers a powerful tool for multi-step or combinatorial synthetic chemistry, a field in which the search for novel drugs ever demands more rapid and efficient methods. Although solid-phase organic synthesis (SPOS) has been of great help [63], several drawbacks still handicap its use, e.g. lengthy reaction times resulting from the use of heterogeneous catalysis. The coupling of RTILs with MW combines a powerful non-conventional heating method with a thermally stable medium that strongly absorbs MW, leading to significant rate enhancements and higher product yields. Moreover, Bazureau and coworkers showed that TSILs with grafted poly(ethyleneglycol) chains could efficiently replace soluble polymeric matrices in combinatorial chemistry [64]. Scheme

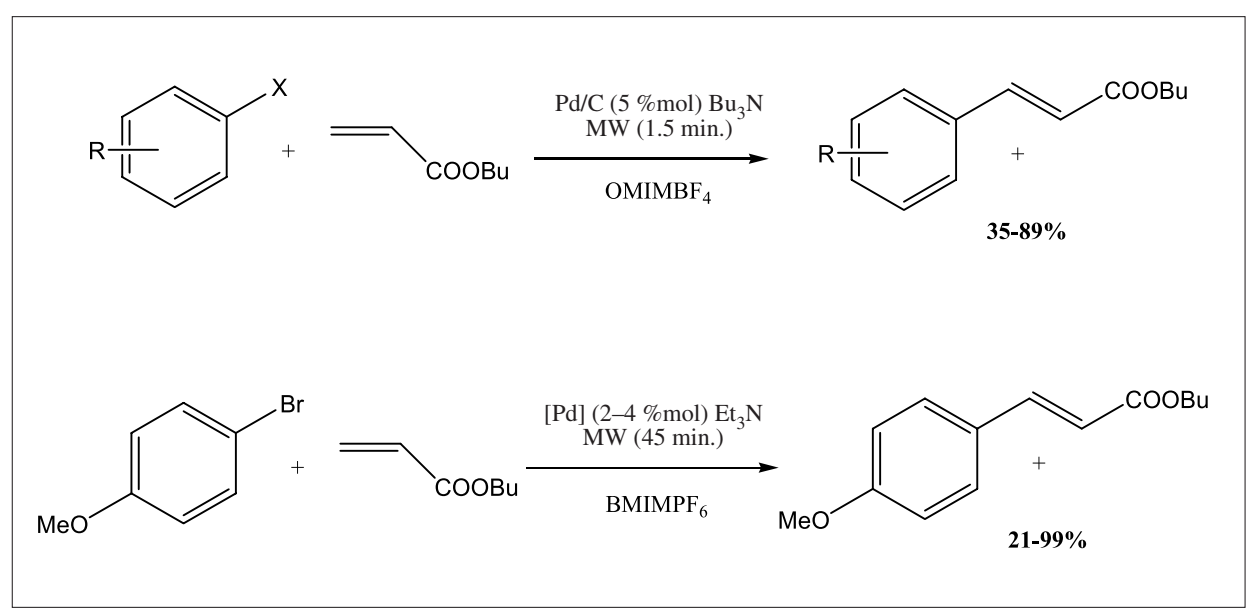

Scheme 6. Heck reaction in RTILs under MW irradiation

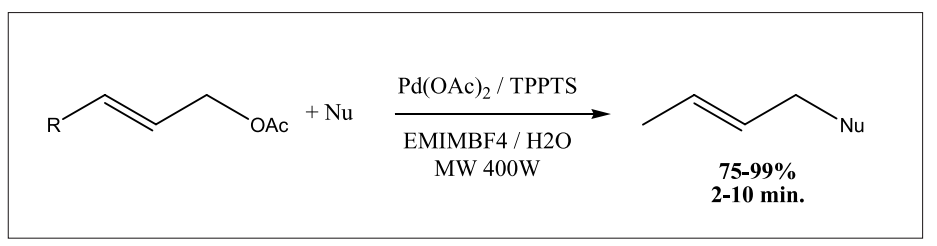

Scheme 7. Trost-Tsuji coupling in RTILs
8 presents the MW-assisted preparation of 2-thioxotetrahydropyridin-4-(1H)-ones, a IoLiPOS (Ionic Liquid Phase Organic Synthesis) procedure replacing the SPOS method [65].

MW-assisted IoLiPOS has also been successfully applied to the synthesis of 4thiazolidinones, 1,4-dihydropyridines, 3,4dihydropyrimidin-2(1H)-ones and polyhydroquinolines [66]. Based on the same principle, a very interesting three-step synthesis (Pictet-Spengler plus Schotten-Baumann and intramolecular ester amidation) has been recently performed to access tetrahydro- $\beta$-carbolinediketopiperazines, belonging to the indole alkaloids [67]. The total re- action time did not exceed $5 \mathrm{~min}$ and yields fell in the range 49-69\% (Scheme 9).

Likewise, a variety of polyfunctionalized 4H-pyrans were prepared, containing a structural unit that is found in a number of biocompounds endowed with interesting pharmacological activities.

\subsubsection{Organic Synthetic Chemistry}

The Diels-Alder reaction is one of the most studied in RTIL media [29]. The highly polar nature of the latter dramatically enhanced conversion yields in numerous instances. Yet some particular cases, notably hetero Diels-Alder cycloadditions, still suffer from long reaction times even at high

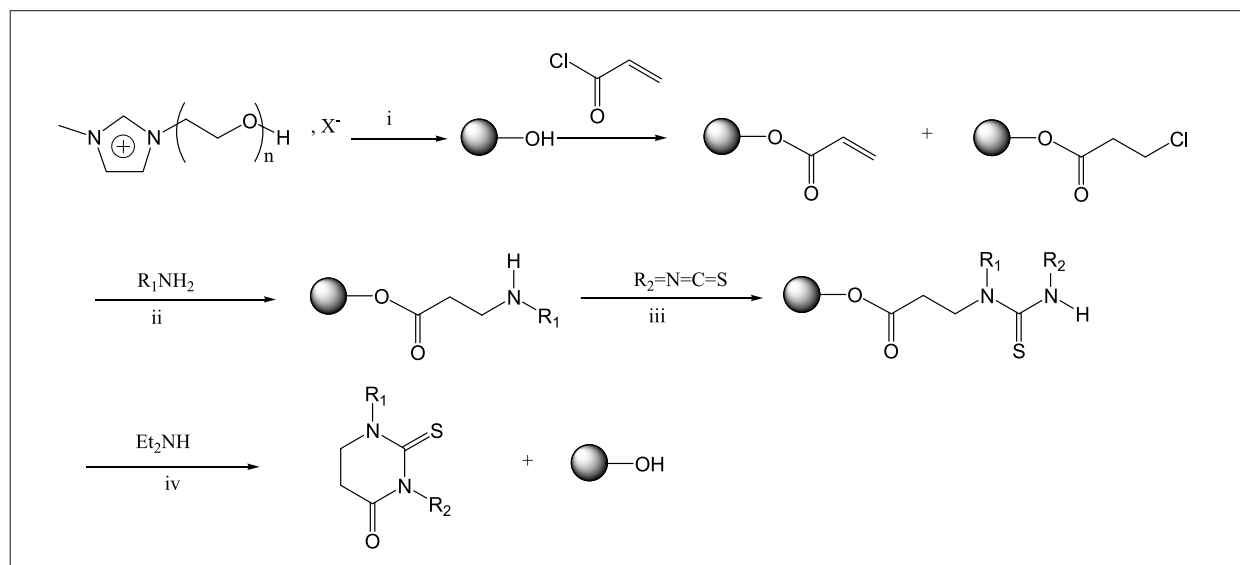

Scheme 8. IoLiPOS method for three-component synthesis. Reagents and reaction conditions: (i) 2 (1.2 equiv.), DCM, reflux, 48 h; (ii) 5 (1 equiv.), MeCN, $25^{\circ} \mathrm{C}, 24$ h; (iii) 7 (1 equiv.), MeCN 18 h; (iv) DEA (2 equiv.), MW, $120^{\circ} \mathrm{C}$ (power 20\%), 15-45 min then purification by flash chromatography on silica gel 60F 254 (Merck). 


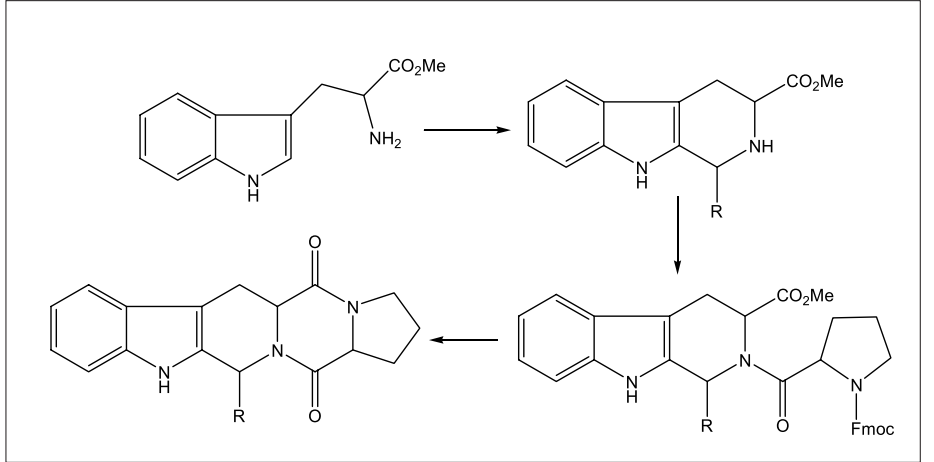

Scheme 9. Three-step synthesis in RTILs under MW irradiation. Synthesis of tetrahydro-b-carbolinediketopiperazines. Reagents and conditions: (1) RCHO (12 equiv.), 10\% TFA, [bdmim] $\left[\mathrm{PF}_{6}\right] /$ THF (1:1), microwave (60 W), 60 ${ }^{\circ} \mathrm{C}, 25 \mathrm{~s}$; (2) Cbz-Pro-Cl (5 equiv.), DIEA (5 equiv.) [bdmim]PF 6 /THF (1:1), rt, $3 \mathrm{~min}$; (3) 20\% piperidine, [bdmim[ $\left.\mathrm{PF}_{6}\right] / \mathrm{THF}$ (1:1), microwave (60 W), 60 ${ }^{\circ} \mathrm{C}, 60 \mathrm{~s}$. temperatures and under pressure. Recently Kappe, Van Der Eycken et al. carried out under MW in biphasic DCE/ionic liquid systems an intramolecular hetero DielsAlder involving a series of alkenyl-tethered 2(1H)-pyrazinones [68]. As the media absorbed MW strongly, they were used in catalytic amounts to prevent any potential side-reaction due excessive or local heating. Reaction times decreased from $2 \mathrm{~d}$ to an average 20 min (Scheme 10).

Many TSILs have been prepared by a MW-assisted method [53-57]. We must emphasize that some of them have been successfully used as 'MW-absorbing agents' to enhance the rates of diverse chemical transformations (Scheme 11).

Two major points should be stressed here. Firstly, even functionalized RTILs are thermally stable under MW; secondly, the versatility of RTILs as green media for various chemical transformations is far greater than that of conventional molecular solvents, while hazards arising with the latter are avoided. But the most exciting development of RTILs is perhaps yet to come. Several groups have tried to saddle them with the dual role of solvent and catalyst, reaping additional advantages from the standpoint of green chemistry. This target

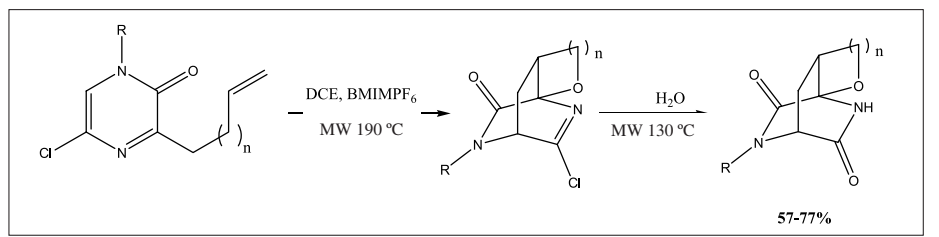

Scheme 10. Intramolecular hetero Diels-Alder reaction in RTILs

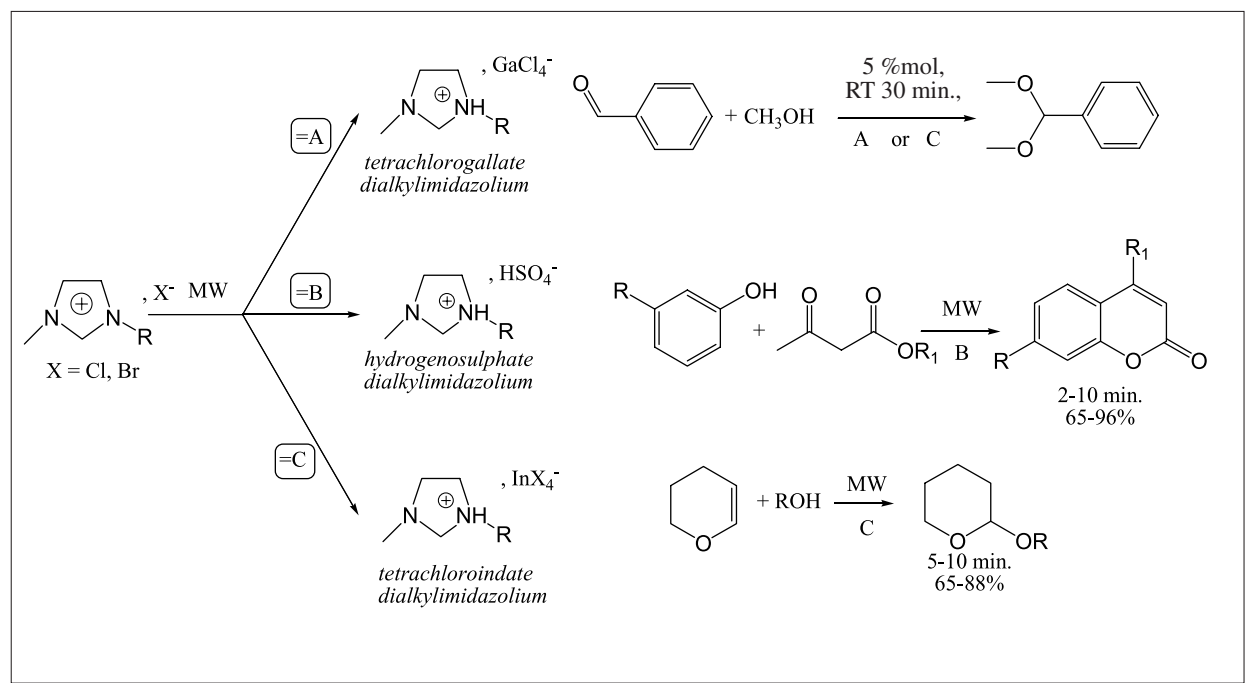

Scheme 11. MW-assisted preparation of TSIL and their use as solvents for chemical transformations can be achieved using a TSIL or sometimes even with a non-functionalized RTIL. Ren and $\mathrm{Wu}$ have showed that simple halide RTILs can act in the dual role of reagent (nucleophile) and solvent in the conversion of primary alcohols to alkyl halides in the presence of an organic or inorganic acid [69]. The main drawback encountered was a lengthy reaction time (24-48 h). Using MW instead Leadbeater et al. obtained spectacular results in terms of improved yields and, above all, of shortened reaction times. These dropped from $24-48 \mathrm{~h}$ to a few minutes [70].

Ranu and Jana performed under MW a stereoselective debromination of vicinal dibromides in an easily accessible ionic liquid, 1-methyl-3-pentylimidazolium fluoroborate [71]. The debromination reaction usually requires either the use of $\mathrm{Sm}, \mathrm{In}$, $\mathrm{Mg}$, or $\mathrm{Zn}$ in a refluxing organic solvent, generally THF or $\mathrm{MeOH}$, or the use of reducing agents such as sodium borohydride or sulfide. In the medium chosen by the authors the reaction proceeded in a well controlled and easy way under MW irradiation without resorting to reducing agents; the released bromine vapors were trapped in a cyclohexene solution (Scheme 12).

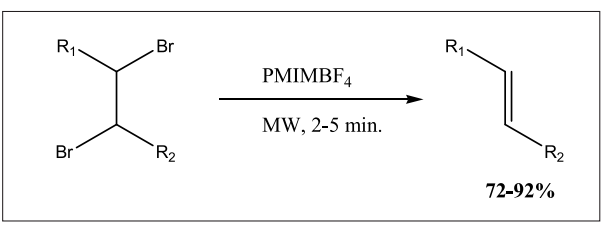

Scheme 12. Debromination of vicinal dibromides under MW in RTILs

Last but not least, the reaction was highly stereoselective, as only trans olefins were obtained. Although other easily accessible RTILs were tested under the same conditions, none of them afforded the expected product. The authors concluded that 1-methyl-3-pentylimidazolium tetrafluoroborate might be involved in the mechanism and postulated a pathway in which the $\mathrm{BF}_{4}$ anion played a major role.

A similar approach was used by $\mathrm{Ba}-$ zureau et al. for the esterification of carboxylic acids with alcohols [72] (Scheme 13). The TSIL employed (1-butyl-3-methylimidazolium hydrogenosulfate BMIMHSO4), also prepared by a MW-assisted method, is a Brönsted acid, while most of the previously available RTILs had been considered to be Lewis acids. The emergence of RTILs that are Brönsted acids is of great interest in organic synthetic chemistry and may enlarge the potential of TSILs as solvents and/or reagents.

Again, the above-cited authors could recycle the ionic liquid and reuse it several times without any loss of activity. 


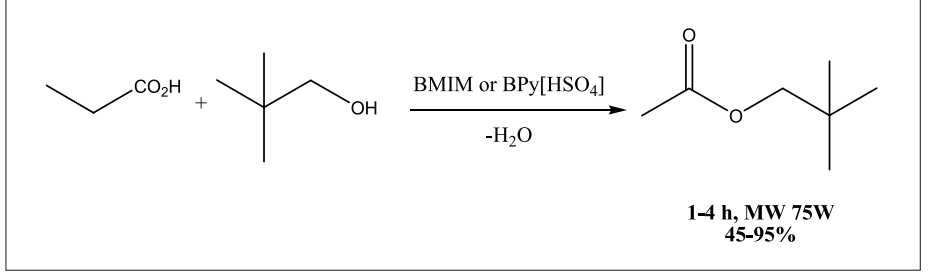

Scheme 13.

While the combination of MW with RTILs offers a very efficient method to enhance various chemical transformations, it must be emphasized that under MW irradiation these compounds can undergo decomposition at temperatures above 200$250{ }^{\circ} \mathrm{C}$ as they do in vacuum pyrolysis in the range $300-600{ }^{\circ} \mathrm{C}$, leading to the same decomposition products. Any planned process should therefore be optimized to circumvent this potential source of side products. With up-to-date MW apparatus, that is equipped with a fine temperature control, decomposition reactions can easily be prevented.

\subsubsection{The Use of RTILs as Green, Recyclable Media in US-assisted Synthesis}

The earliest reports on the combination of MW with RTILs are contemporary to the first ones concerning the combination US/ionic liquids. Owing to the high absorbance of ionic compounds, MW has rapidly overcome US in this context. In fact the two techniques do differ in the ways they activate reactants. MW is a rapid thermal activation method suitable for polar systems, particularly ionic liquids. US, by cavitation phenomena, may induce specific chemical effects resulting from the generation of radical intermediate species as well as physical effects like micro-emulsion, mass transport and reduction of particle size. The relatively high viscosities and densities of RTILs can be considered as factors opposing cavitation as well as reducing the intensity of physical effects. On the other hand, it is now clear that the lower the vapor pressure of a compound, the lower its tendency to enter cavitation bubbles and so to encounter the high temperatures and pressures arising from bubble collapse. Then ionic liquids, when used as solvents under ultrasonic irradiation, should not suffer decomposition because of cavitation phenomena. On the contrary, organic compounds having higher vapor pressures, when dissolved in ionic liquids, will enter the bubbles and meet the extreme conditions accompanying bubble collapse. This could explain at least in part the enhanced reactivity of organic compounds.

In fact even RTILs suffer decomposition when irradiated alone with US. Suslick and coworkers have recently screened several RTILs and found that all of them were decomposed by low-frequency irradiation yielding their respective starting materials [73]. The authors insisted that this occurred at the second site of reaction under ultrasonic irradiation, i.e. the surrounding shell. They measured $1900 \mathrm{~K}$ at this gas-liquid interface, a temperature much above the thermal degradation threshold for ionic liquids. They also emphasized that cavitation phenomena heavily depend on the chemical properties of the fluid medium. This is in accordance with the hypothesis that organic solutes having higher vapor pressures will preferentially enter cavitation bubbles, providing an interesting approach to controlling the chemical composition of the gaseous phase in the bubbles. Obviously, their decomposition strongly depends on the acoustic energy released into the system. In an ultrasonic bath or with piezoelectric ceramic (frequencies above $200 \mathrm{kHz}$ ), decomposition of an ionic liquid should be almost negligible. On the contrary, the high energy output of an ultrasonic probe at 20 $\mathrm{kHz}$ should lead to a noticeable decomposition level.

The use of RTILs as solvents under ultrasonic irradiation has been exploited for various chemical reactions, among them the para-nitration of phenols [74], Suzuki and Heck $\mathrm{C}-\mathrm{C}$ couplings [75][76], the synthesis of some methanofullerene derivatives [77], and Diels-Alder cycloadditions [78]. In all cited cases, the combination US/RTILs improved the process by cutting down reaction times while increasing yields. However, none of the authors, while reporting successful recycling of the media, discussed their potential decomposition.

\section{Acknowledgments}

This work has been carried out under the auspices of the European Union COST Action D32 (Working Group D32/006/04: Microwave and high-intensity ultrasound in the synthesis of fine chemicals).

Received: March 24, 2006

[1] M. Maeda, H. Amemiya, New J. Chem. 1995, 19, 1023.

[2] G. Cravotto, S. Di Carlo, V. Tumiatti, C. Roggero, D. Bremner, Environ. Technol. 2005, 26, 721 .
[3] A. Cartellieri, P.H. Thiesen, B. Niemeyer, Waste Management 2005, 25, 985.

[4] M.D. Luque de Castro, M.M. JimènezCarmona, V. Fernandez-Pèrez, Trends in Anal. Chem. 1999, 18, 708 .

[5] W.S. Chu, Q. Liang, J.L. Liu, M.Q. Wei, M. Winters, L. Liotta, G. Sandberg, M.K. Gong, Lab. Investig. 2005, 85, 1416.

[6] R. Sanghi, S.S. Kannamkumarath, J. Anal. Chem. 2004, 59, 1032.

[7] Y. Mastai, A. Gedanken, in 'Chemistry of Nanomaterials' Ed. C.N.R. Rao, A. Mueller, A.K. Cheetham, 1, 113. Wiley-VCH, 2004.

[8] C.O. Kappe, Angew. Chem. Int. Ed. 2004 43, 6250.

[9] L. Perreux, A. Loupy, 'Microwaves in Organic Synthesis', Ed. A. Loupy, Wiley$\mathrm{VCH}, 2002$.

[10] G. Cravotto, P. Cintas, Chem. Soc. Rev. 2006, 35, 180 .

[11] B. Toukoniitty, J.P. Mikkola, D.Y. Murzin, T. Salmi, Appl. Catal. A 2005, 279, 1.

[12] G. Cravotto, L. Boffa, M. Turello, M. Parenti, A. Barge, Steroids 2005, 70, 77.

[13] W. Bonrath, Ultrason. Sonochem. 2004, 11,1 .

[14] V. Roy, L. Colombeau, R. Zerrouki, P. Krausz, Carbohydr. Res. 2004, 339, 1829.

[15] M. Nuchter, B. Ondruschka, W. Bonrath, A. Gum, Green Chem. 2004, 6, 128.

[16] S.E. Wolkenberg, W.D. Shipe, C.W. Lindsley, J.P. Guare, J.M. Pawluczyk, Curr. Opin. Drug Disc. \& Devel. 2005, 8, 701.

[17] T.J. Mason, D. Peters, 'Practical Sonochemistry. Power Ultrasound: Uses and Applications', Ellis Horwood, Chichester, 2002

[18] T.J. Mason, J. Lorimer, 'Applied Sonochemistry: Uses of Power Ultrasound in Chemistry and Processing', Wiley-VCH, 2002.

[19] A. Lagha, S. Chemat, P.V. Bartels, F. Chemat, Analusis 1999, 27, 452.

[20] Y. Peng, G. Song, Green Chem. 2001, 3, 302.

[21] Y. Peng, G. Song, Green Chem. 2002, 4, 349.

[22] Y. Peng, G. Song, Green Chem. 2003, 5, 704.

[23] F. Chemat, M. Poux, J.L. Di Martino, J. Berlan, J. Microw. Power Electrom. En. 1996, 31, 19.

[24] F. Chemat, M. Poux, S.A. Galema, $J$. Chem. Soc., Perkin Trans. 2 1997, 2371.

[25] S. Chemat, A. Lagha, H.A. Amar, F. Chemat, Ultrason. Sonochem. 2004, 11, 5 .

[26] G. Cravotto, M. Beggiato, G. Palmisano, J.M. Lévêque, W. Bonrath, Tetrahedron Lett. 2005, 46, 2267.

[27] Y. Peng, R. Dou, G. Song, J. Jiang, Synlett 2005, 14, 2245.

[28] P. Wasserscheid, T. Welton, 'Ionic liquids in synthesis', Wiley-VCH, 2002.

[29] D.J.A.C.E. Tucker, Tet. Lett. 1989, 30, 1785; J. Howarth, K.H.D. Fayne, P. McCormac, Tet. Lett. 1997, 38, 3097; C.W. 
Lee, Tet. Lett. 1999, 40, 2461; T. Fischer, A.S.T. Welton, J. Woolf, Tet. Lett. 1999, 40, 793; F.Z.A.T Kitazume, Green Chem. 2000, 2, 137; A.P. Abbott, G.C.D.L. Davies, R.K. Rasheed, V. Tambyrajah, Green Chem. 2002, 4, 24.

[30] A. Starck, B.L. MacLean, R.D. Singer, $J$. Chem. Soc., Dalton Trans. 1999, 63; S.J. Nara, J.R. Harjani, M. Salunkhe, J. Org. Chem. 2001, 66, 8616; Z. Zhao, Z.L.G. Wang, W. Qiao, L. Cheng, Appl. Catal. A 2004, 262, 69.

[31] W.A. Herrmann, V.P.W. Böhm, J. Organometal. Chem. 1999, 572, 141; A. J. Carmichael, M.J. Earle, J.D. Holbrey, P.B. McCormac, K.R. Seddon, Org. Lett. 1999, 7, 997; L. Xu, W. Chen, J. Xiao, Organometal. 2000, 19, 1123; B.M. Choudary, S.M. Naidu, S. Chowdari, M.L. Kantam, B. Sreedhar, J. Am. Chem. Soc. 2002, 124, 14127; F. Berthiol, M. Feuerstein, H. Doucet, M. Santelli, Tet. Lett. 2002, 43, 5625; G. Zou, Z. Wang, J. Zhu, J. Tang, M. H. He, J. Mol. Catal. A: Chem. 2003, 206, 193; S.A. Forsyth, H.Q.N. Gunaratne, C. Hardacre, A. McKeown, D.W. Rooney, K.R. Seddon, J. Mol. Catal. A: Chem. 2005, 231, 61.

[32] B.M. Choudary, S.M. Naidu, S. Chowdari, M.L. Kantam, B. Sreedhar, J. Am. Chem. Soc. 2002, 124, 14127; G. Zou, Z. Wang, J. Zhu, J. Tang, M.H. He, J. Mol. Catal. A: Chem. 2003, 206, 193; C.J. Mathews, P.J. Smith, T. Welton, J. Mol. Catal. A: Chem. 2004, 214, 27; A. Corma, H. Garcia, A. Leyva, Tetrahedron 2005, 61, 9848.

[33] S.-L. Chen, S.-J. Ji, T.-P. Loh, Tet. Lett. 2003, 44, 2405; X.-F. Yang, M. Wang, R.S. Varma, C.-J. Li, J. Mol. Catal. A: Chem. 2004, 214, 147; S. Sahoo, T. Joseph, S.B. Halligudi, J. Mol. Catal. A: Chem. 2005, 244, 179.

[34] M.M. Dell'Anna, P. Mastrorilli, C.F. Nobile, G. Romanazzi, G.-P. Suranna, Chem. Commun. 2002, 434; R.T. Dere, R.R. Pal, P.S. Patil, M.M. Salunkhe, Tet. Lett. 2003, 44, 5351; V. Gallo, P. Mastrorilli, C.F. Nobile, G.-P. Suranna, Y. Wang, J. Organometal. Chem. 2005, 690, 3535.

[35] J. Howarth, Tet. Lett. 2000, 41, 6627; R. Bernini, A. Coratti, G. Fabrizi, A.Goggiamani, Tet. Lett. 2003, 44, 8991; R. Bernini, E. Mincione, A. Coratti, G. Fabrizi, G.Battistuzzi, Tetrahedron 2004, 60, 967; A. Ansari, S. Joyasawal, M.K. Gupta, J.S. Yadav, R. Gree, Tet. Lett. 2005, 46, 7507.

[36] Y. Chauvin, L. Mussmann, H. Olivier, Angew. Chem. Int. Ed. Engl. 1995, 34, 2698; P.A.Z. Suarez, J.E.L. Dullius, S. Einloft, R.F. De Souza, J. Dupont, Polyhedron 1996, 15, 1217; R.A. Brown, E. Mckoon, C.A. Eckert, C.L. Liotta, P.G. Jessop, J. Am. Chem. Soc. 2001, 123, 1254; J. Dupont, G.S. Fonseca, A.P. Umpierre, P.F.P Fichtner, S.R. Teixeira, J. Am. Chem. Soc. 2002, 124, 4228; P.J. Dyson, D.J. Ellis, W. Henderson, G. Laurenczy, Adv. Synth. Ca- tal. 2003, 345, 216; D.-Q. Xu, Z.-Y. Hu, W.-W. Li, S.-P. Luo, Z.-Y. Wu, J. Mol. Catal. A: Chem. 2005, 235, 137.

[37] A.E. Visser, R.P. Swatloski, R.D. Rogers, Green Chem. 2000, 1, 1; A.E. Visser, R.P. Swatloski, W.M. Reichert, R. Mayton, S. Sheff, A. Wierzbicki, J.H. Davis, R.D. Rogers, Chem. Commun. 2001, 135.

[38] R.P. Swatlosky, S.K. Spear, J.D. Holbrey, R.D. Rogers, J. Am. Chem. Soc. 2002, 124 4974; Y. NuLi, J.Yang, R. Wu, Electrochem. Commun. 2005, 7, 1105.

[39] M.T. Reetz, W. Wiesenhöfer, G. Francio, W. Leitner, Chem. Commun. 2002, 992; M. Persson, U.T Bornscheuer, J. Mol. Catal. B: Enzym. 2003, 22, 21; N. Jain, A. Kumar, S. Chauhan, S.M.S. Chauhan, Tetrahedron 2005, 61, 1015.

[40] A.J. Carmichael, D.M. Haddleton, S.A.F. Bon, K.R. Seddon, Chem. Commun. 2000 , 1237; S. Perrier, T.P. Davis, A.J. Carmichael, D.M. Haddleton, Eur. Polymer J. 2003, 39, 417.

[41] N. Papageorgiou, M. Armand, P. Bonhôte, H. Pettersson, A. Azam, M. Grätzel, J. Electrochem. Soc. 1996, 143, 3099.

[42] P. Wasserscheid, T. Welton, 'Ionic liquids in synthesis', Wiley-VCH, Weinheim, 2002; T. Welton, Coordination Chem. Rev. 2004, 248, 2459.

[43] C. Baudequin, J. Baudoux, J. Levillain, D. Cahard, A.-C. Gaumont, J.-C. Plaquevent, Tet. Asym. 2003, 14, 3081.

[44] P. Wasserschied, A. Bösmann, C. Bolm, Chem. Commun. 2002, 200; Z. Wang, Q. Wang, Y. Zhang, W. Bao, Tet. Lett. 2005, 46, 4657.

[45] N.Z. Menschutkin, Phys. Chem. 1890, 6, 41; J.A. Zoltewicz, L.W. Deady, Adv. Heterocycl. Chem. 1978, 22, 71.

[46] R. Bohlmann, in 'Comprehensive Organic Synthesis', Ed. B.M. Trost, I. Fleming, Pergamon Press, Oxford, 1991, vol. 6, pp. 203.

[47] R.A. Carpio, L.A. King, R.E. Lindstrom, J.C. Nardi, C.L. Hussey, J. Electrochem. Soc.: Electrochem. Sci. Technol. 1979, 126, 1644; J.S. Wilkes, J.A. Levisky, A.W. Robert, C.L. Hussy, Inorg. Chem. 1982 21, 1263.

[48] L. Perreux A. Loupy, 'Microwaves in Organic Synthesis', Ed. A. Loupy, Wiley$\mathrm{VCH}$, Weinheim, 2002.

[49] R.S. Varma, V.V. Namboodiri, Chem. Commun. 2001, 643.

[50] B. M. Khadilkar, G.L. Rebeiro, Org. Proc. Res. \& Dev. 2002, 6, 826.

[51] M. Deetlefs, K.R. Seddon, Green Chem. 2003, 5, 181.

[52] V.V. Namboodiri, R.S. Varma, Tet. Lett. 2002, 43, 5381.

[53] V.V. Namboodiri, R.S. Varma, Chem. Commun. 2002, 916.

[54] V.V. Namboodiri, R.S. Varma, Chem. Commun. 2002, 916; Y.J. Kim, R.S. Varma, Tet. Lett. 2005, 46, 7447.

[55] Y.J. Kim, R.S. Varma, Tet. Lett. 2005, 46, 1467.
[56] V. Singh, S. Kaur, V. Sapehiyia, J. Singh, G.L. Kad, Cat. Commun. 2005, 6, 57.

[57] G. Vo Thanh, B. Pegot, A. Loupy, Eur. J. Org. Chem. 2004, 1112.

[58] V.V. Namboodiri, R.S. Varma, Org. Lett. 2002, 4, 3161 .

[59] J.M. Lévêque, S. Desset, J. Suptil, C. Fachinger, M. Draye, W. Bonrath. G. Cravotto, Ultrason. Sonochem. 2006, 13, 189.

[60] J.M. Lévêque, J.L. Luche, C. Pétrier, R. Roux, W. Bonrath, Green Chem. 2002, 4, 357.

[61] K.S.A. Vallin, P. Emilsson, M. Larhed, A. Hallberg, J. Org. Chem. 2002, 67, 6243; X. Xie, J. Lu, B. Chen, J. Han, X. She, X Pan, Tet. Lett. 2004, 45, 809.

[62] M.-C. Liao, X.-H. Duan, Y.-M. Liang, Tet. Lett. 2005, 46, 3469.

[63] Z.F. Dörwald in 'Organic Synthesis on Solid Phase', Wiley-VCH, Weinheim, 2000.

[64] J. Fraga-Dubreuil, J.-P. Bazureau, Tetrahedron 2003, 59, 6121.

[65] H. Hakkou, J.-J. VanDen Eynde, J. Hamelin, J.-P. Bazureau, Tetrahedron 2004, 60 , 3745.

[66] J.-J. Legeay, J.-J. Vanden Eynde, J.-P. Bazureau, Tetrahedron 2005, 61, 12386; F Yi, Y. Peng, G. Song, Tet. Lett. 2005, 46, 3931.

[67] Y.-H. Yen, Y.-H. Chu, Tet. Lett. 2004, 45, 8137.

[68] E. Van Der Eycken, P. Appukkuttan, W. De Borggraeve, W. Dehaen, D. Dallinger, C.O. Kappe, J. Org. Chem. 2002, 67, 7904.

[69] R.X. Ren, J.X. Wu, Org. Lett. 2001, 3, 3727; R.X. Ren, J.X. Wu, Tet. Lett. 2002, 43, 387.

[70] N.E. Leadbeater, H. M. Torenius, H. Tye, Tetrahedron 2003, 59, 2253.

[71] B.C. Ranu, R. Jana, J. Org. Chem. 2005 , 70,8621

[72] J. Fraga-Dubreuil, K. Bourahla, M. Rahmouni, J.-P. Bazureau, J. Hamelin, Catal. Commun. 2002, 3, 185.

[73] D.J. Falnnigan, S.D. Hopkins, K.S. Suslick, J. Organometal. Chem. 2005, 690, 3513.

[74] R. Rajagopal, K.V. Srinivasan, Ultrason. Sonochem. 2003, 10, 41.

[75] R. Rajagopal, D.V. Jarijote, K.V. Srinivasan, Chem. Commun. 2002, 616.

[76] R. Rajagopal, D.V. Jarijote, K.V. Srinivasan, Chem. Commun. 2001, 1544.

[77] Z. Yingghuai, J. Phys. Chem. Solids 2004, $65,349$.

[78] J.L. Bravo, I. Lopez, P. Cintas, G. Dilvero, M.J.Arévalo, Ultrason. Sonochem. in press DOI:10.1016/j.ultsonch.2005.07.001. 